\title{
Assessing Collision Algorithms for the NewSpace Era
}

\author{
Mr. Samuel Diserens ${ }^{(1)}$, Professor Hugh G. Lewis ${ }^{(2)}$, and Professor Joerg Fliege ${ }^{(3)}$ \\ (1) University of Southampton, Boldrewood Innovation Campus, Burgess Rd, Southampton SO16 7QF, UK, \\ S.D.Diserens@Soton.ac.uk \\ (2) University of Southampton, Boldrewood Innovation Campus, Burgess Rd, Southampton SO16 7QF, UK, \\ H.G.Lewis@Soton.ac.uk \\ (3) University of Southampton, Highfield, University Rd, Southampton SO17 1BJ, UK, \\ J.Fliege@Soton.ac.uk
}

\begin{abstract}
The 'NewSpace' era of the last two decades has seen increasing disruption and innovation in the space industry driven by a growing commercial element. A new investigation of spacecraft launched between 1980 and 2017 identified an increase in the number of spacecraft being deployed into similar target orbits. This has resulted in a shift from a more random spatial distribution of objects to a situation with greater spatial structure. With collisions expected to be an increasing and leading source of space debris it is important that we understand the performance of collision models and their sensitivity to the changing environment. We believe that current collision algorithms have the potential to introduce errors in the estimation of collision probabilities when modelling NewSpace scenarios. Two probabilistic algorithms, the Cube approach (Liou et al., 2003) and the Orbit Trace method (based on Öpik, 1951) were investigated for a range of scenarios.

An implementation of the Cube algorithm was verified using the original Jovian moons case and highlighted issues relating to convergence of the average collision probability for a pair of objects and to the relationship between the collision probability and the size of the cube used. Further tests compared the collision probabilities generated by the Satellite Orbital Conjunction Reports Assessing Threatening Encounters in Space (SOCRATES) against those calculated using each of the Cube and Orbit Trace algorithms in conjunction with the SGP4 propagator. Simulations of specific sub-sets of the tracked populations for well-defined time periods showed substantial discrepancies between the different collision methods.

The results suggest some inaccuracies in the currently used collision methods, introducing additional uncertainty to the rate of population growth in simulations of the evolution of the debris environment. Further work is ongoing to investigate the relationship between this variance and spacecraft altitude to ascertain whether current models are accurately representing where fragmentations might occur.
\end{abstract}

\section{INTRODUCTION}

Artificial space debris is widely acknowledged to pose a risk to the safe operation of both crewed and uncrewed spacecraft in near earth orbit. As of January 2019 there were believed to be over 900,000 objects in orbit with a size greater than $1 \mathrm{~cm}$ and the potential to fatally damage a spacecraft. Of these objects only around 34,000 were big enough $(>10 \mathrm{~cm})$ to be regularly tracked [1]. A number of computational models [2] have been used to perform simulations of the future debris environment in order to better understand how this debris population might develop. Some of the initial studies of this environment by Kessler and Cour-Palais [3], and by Kessler and Anz-Meador [4] postulated that there exists a critical density of debris objects above which random collisions among the objects will cause the population to become self-sustaining and possibly lead to runaway growth.

Since the year 2000 there has been a greater than twelve-fold increase in the average number of space investors per year [5] reflecting a significant increase in the amount of commercial involvement in the space industry. The shift towards greater private sector participation, as opposed to the traditional operators from governments and the military-industrial complex, has led to increased competition. This competition has driven developments and trends in spacecraft missions and technologies such as an increase in smaller, lighter satellites, novel methods like additive manufacturing, and mass launch events such as the PSLV-C37 mission [6].

These changes - generally grouped under the banner of 'NewSpace' or 'the new space age' [7] - have the potential to considerably impact the future evolution of the debris environment. As such, it is important to assess whether these changes affect the validity of the debris models being used to understand the potential future environment. 


\section{STUDYING NEWSPACE TRENDS}

Key characteristics, both orbital and physical, were analysed for spacecraft launched between 1980 and 2019 using data extracted from the European Space Agency's (ESA) Database and Information System Characterising Objects in Space (DISCOS). To study how the composition of the spacecraft population had changed the records for all spacecraft from those in Low Earth Orbit (LEO) to those in geosynchronous equatorial orbit (GEO) and above were sorted into categories of military, civil, academic and commercial in accordance with the nature of the operating organization. Figure 1 compares the prevalence of these categories for recent decades. The growth in the proportion of commercial spacecraft can be seen, with an increase from $4.6 \%$ of the launch population in the 1980 s to $55.6 \%$ in the most recent decade. Over the same period, the proportion of military spacecraft in the population has reduced from $76.5 \%$ to only $10.5 \%$.

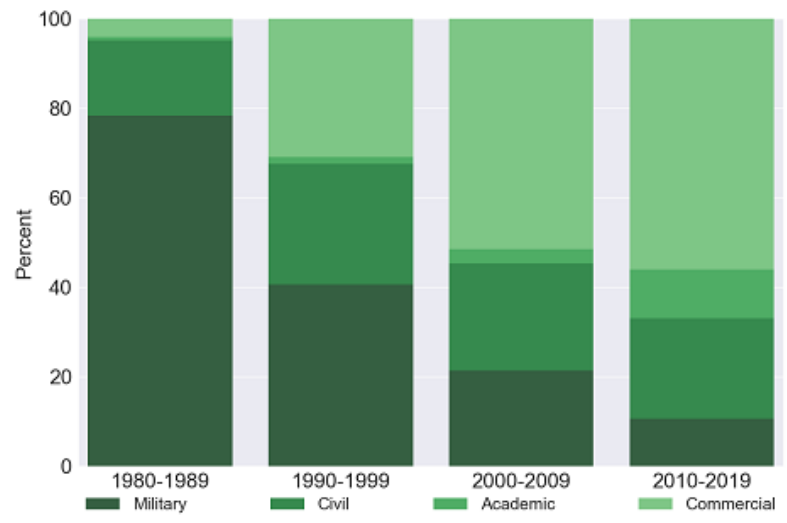

Fig. 1 Percentage of spacecraft launched per decade by organisation type.

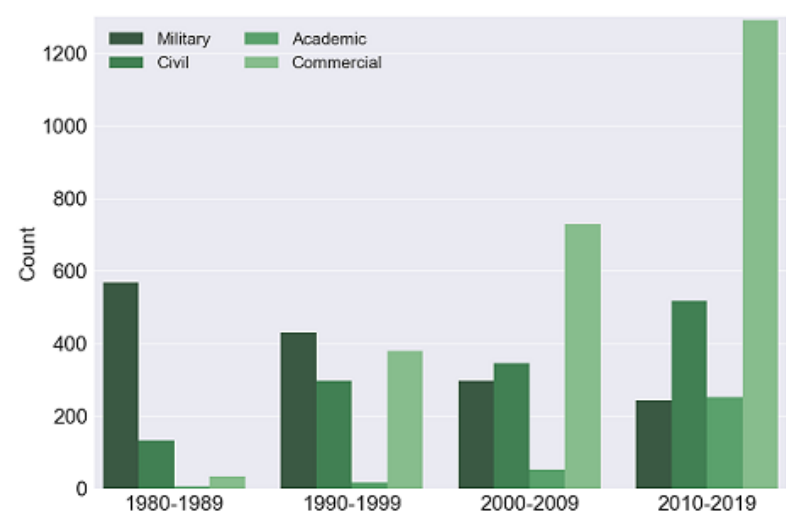

Fig. 2 Count of spacecraft launched per decade by organisation type.

While this military decrease can be attributed in part to the dissolution of the USSR and the end of the Cold War in the early 1990s the absolute launch numbers, shown in Fig. 2, indicate that this is not the sole cause. Total launches for civil and military spacecraft has remained consistent at around 700 per decade, but has been accompanied by a $213 \%$ increase in overall launches from the 1980s to the 2010s. Commercial and academic spacecraft account for 208\% of this increase, from 34 to 1292 and 5 to 253 launched respectively.

As well as innovations in spacecraft construction in recent years, there have also been changes in operational characteristics, including a growing trend towards ridesharing and the launch of multiple spacecraft on the same rocket into similar orbits. One result of the increasing commercial presence is that the number of spacecraft operators has increased, from an average of 18 different organisations launching spacecraft per year in the 1980s to 102 each year for the last decade and 167 in 2017. Further change is expected as companies seek new markets with novel mission types being developed, including on-orbit servicing, end-of-life management and Active Debris Removal.

To study the impact of these changes on the spatial distribution of spacecraft the orbital characteristics of the launch population were analysed over the period for the LEO region. The resulting distributions across semi-major axis and inclination are displayed in Fig. 3 (a - d) for each decade. One clear change that emerges from this is an increase in the amount of clustering of spacecraft into specific regions of altitude and inclination. In Fig 3 (d), showing the distribution for the period from 2010 to 2019, there are clear concentrations of spacecraft in two inclinations: at 50$55^{\circ}$ (the inclination of the ISS) and $95-100^{\circ}$ (for sun-synchronous orbits). In addition to the inclination banding the figure shows that spacecraft are becoming increasingly concentrated in the lower altitudes, with the majority of spacecraft having altitudes under $900 \mathrm{~km}$ (equivalent to a semi-major axis less than $7278 \mathrm{~km}$ ).

The first two Starlink deployments exemplified the practice of multi-spacecraft launches with each consisting of 60 spacecraft on a single Falcon 9 [8]. Large constellations such as Starlink and those planned by OneWeb and Boeing will result in new regions of orbital clustering. SpaceX alone plan on up to 24 Starlink launches in 2020, resulting in a further 1440 spacecraft in similar orbits. 


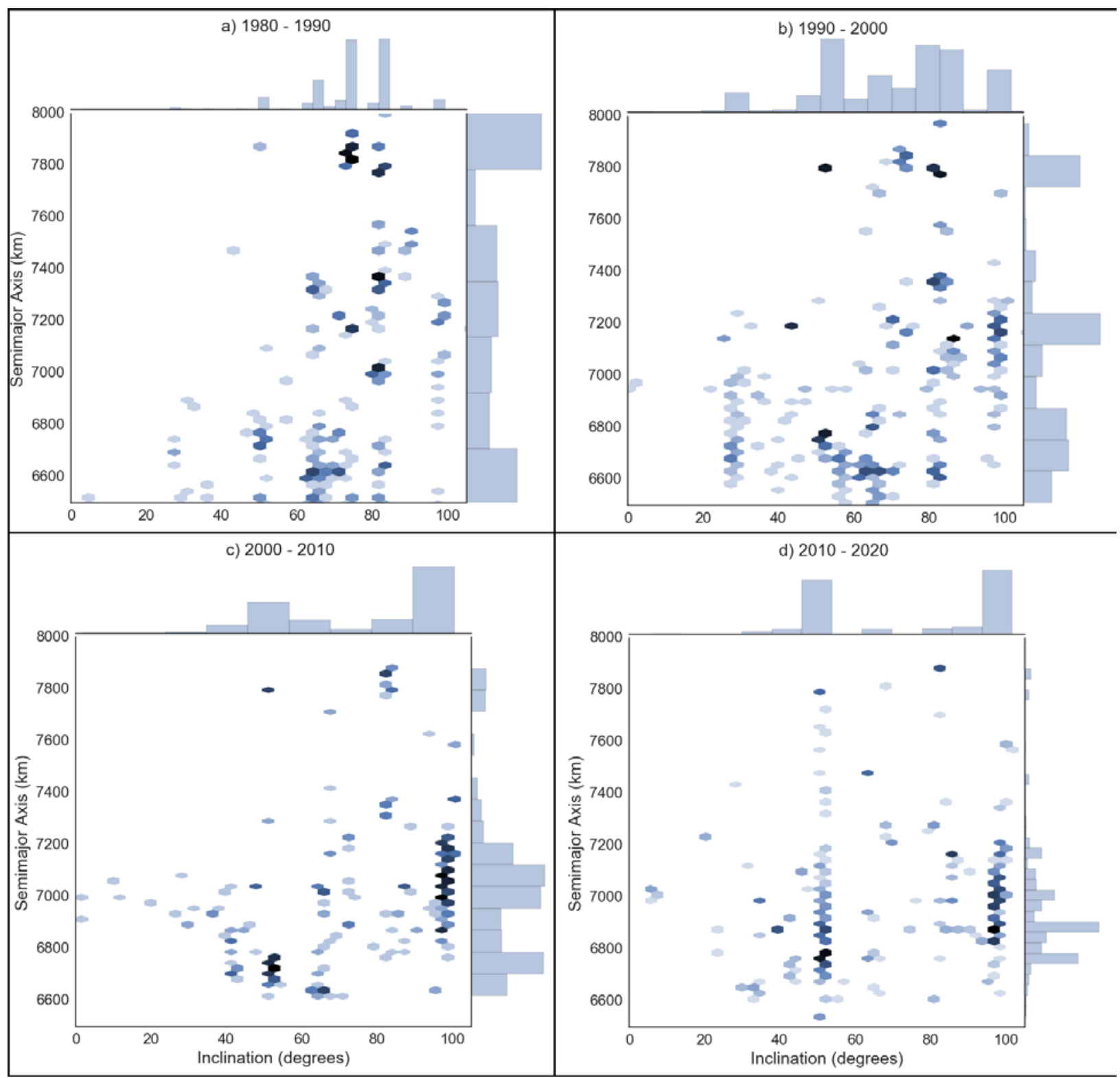

Fig. 3 Plots showing individual histograms and combined 2D hex plots of the distribution of LEO spacecraft across semi-major axis and inclination for (a) 1980-1990; (b) 1990-2000; (c)2000-2010; and (d)2010-2019.

\section{NEWSPACE AND DEBRIS MODELLING}

The evolution of the space debris environment can be broken down into several key processes such as collision, fragmentation, atmospheric drag, which have different impacts on the environment. Table 1 describes the roles that these processes play in the changing environment as sources of new debris objects, sinks for the removal of objects or forces for the migration of objects between regions. The table also presents examples of some of the common component models used to represent these processes in simulations of the environment.

The application of these component models is influenced by the characteristics of debris objects, such as their crosssectional area when calculating collision probabilities or applying atmospheric drag. Assumptions are made in many of these models about these characteristics and how they can be approximated. In light of the on-going changes identified in the physical and orbital characteristics of objects and in the environment it is important to re-asses these assumptions to determine if they are appropriate for the NewSpace era. 
Table 1. Key components of debris models

\begin{tabular}{lll}
\hline Process & Role & Example Model \\
\hline Launch Rate & Source & Historical data \\
Fragmentation & Source & NASA Standard Breakup Model [9] \\
Explosion Rate & Sink & Historic data \\
Collision Prediction & Sink & $\begin{array}{l}\text { Cube [10] } \\
\text { Orbit-Trace [11] }\end{array}$ \\
Atmospheric Decay & Sink & Orbital Propagators \\
\hline \hline Orbital Propagation & Movement & SGP4 [12] \\
\hline \multicolumn{1}{c}{ Atmospheric Drag } & & \\
\multicolumn{2}{l}{ Solar Radiation Pressure } \\
Gravitational Influence (Earth/Moon/Sun)
\end{tabular}

The generation of new debris objects has historically been dominated by the explosion of spacecraft, with these events accounting for $53 \%$ of the total catalogued population and $65 \%$ of the growth in the catalogue between 2008 and 2018 [13]. Accidental collisions between orbiting objects account for only $2.5 \%$ of fragmentation events but contributed $11.6 \%$ of the catalogued debris objects. This difference between proportion of events and proportion of debris produced indicates that, while historically less common, even infrequent collisions could be significant to the future evolution of the debris environment. The data in [13] indicate that the rate of explosions has remained constant since the early seventies while the population of spacecraft has increased exponentially. It is expected that the increasing populations of both spacecraft and debris will result in an increased risk of collision events in future. The combination of these two factors may result in collision events, instead of explosions, becoming the dominant source of new debris objects [3, 4].

Due to the importance of collision events to the future growth of the debris population, this investigation chose to focus on an assessment of the algorithms used to predict collisions within computational debris models. Two key algorithms, Cube and Orbit Trace, were identified as being used in the majority of current debris models [2].

\subsection{The Cube Algorithm}

The Cube algorithm provides a fast, scalable method for identifying collisions within long-term evolutionary models and so has become one of the most commonly used approaches for identifying potential collisions. The total number of collisions $N_{\text {tot }}$ between a pair of objects $i$ and $j$ is estimated by uniformly sampling the collision rate for a pair of objects, $P_{i, j}$, over the simulation projection period.

$$
N_{\text {tot }}=\int_{t_{\text {begin }}}^{t_{\text {end }}} P_{i, j}(t) d t=\int_{s=0}^{s=L}\left[t_{s+1}-t_{s}\right] P_{i, j}(s) d s
$$

When length of the time-steps between these samples, $\left[t_{s+1}-t_{s}\right]$, is sufficiently short the collision rate can be considered constant. This enables the simplification in Eq. 1 where $N_{\text {tot }}$ becomes the integral over all of the timesteps of the collision rate at each step, multiplied by the length of the time-step.

Collisions are considered to be possible in the Cube algorithm only if two objects appear within the same small volume of space, normally a cube. The collision rate at each step is based on the probability that two objects occupy the same cube, and the collision rate for a pair of objects in the same cube, $P_{i, j}$.

The first of these is estimated by sampling the instantaneous positions of every object at each time-step, normally by sampling uniformly from the mean anomaly to select a random point on the object's orbit. The positions at this time are then compared to determine which regions of space are occupied by more than one object.

The collision rate for pairs of co-located objects is calculated using the kinetic theory of gas on the scale of the cube:

$$
P_{i, j}=S_{i} S_{j} V_{r e l} \sigma d U
$$


where $V_{\text {rel }}$ is the relative velocity between the two objects and $d U$ is the volume of the cube. The spatial densities of objects $i$ and $j, S_{i}$ and $S_{j}$ can be considered $1 / d U$ for co-located objects. The collision cross-sectional area: $\sigma=$ $\pi\left(r_{i}+r_{j}\right)^{2}$, where $r_{i}$ and $r_{j}$ are the hard body radii of the objects.

A cube size of $1 \%$ or less of the average semi-major axis of the objects in a system is considered to be small enough to capture the true collision nature of the system. For simulations of the space debris environment a cube with side length of $10 \mathrm{~km}$ is normally used, roughly $0.15 \%$ of the semi-major axis of the lowest objects in LEO.

\subsection{Orbit Trace}

As with the Cube, the Orbit Trace method generates a probability of a collision occurring within a time-step. However, this is done in a pair-wise geometric fashion, looking at the intersection points of the orbit paths using methods originally formed for investigating potential collisions between asteroids and other celestial bodies [14, 15]. The collision probability for a pair of objects is calculated based on the proportion of the time-step each object spends in the region around the intersection of the orbits.

This investigation used an equivalent approach derived by JeongAhn and Malhotra [16] in their equation (13) to calculate this probability:

$$
P_{i, j}=\frac{\pi\left(r_{i}+r_{j}\right) V_{r e l}}{2\left|v_{i} \times v_{j}\right| T_{u, i} T_{u, j}}
$$

Where $r_{i}$ and $r_{j}$ are the hard body radii of the objects, $v_{i}$ and $v_{j}$ are their velocities, $T_{u, i}$ and $T_{u, j}$ are the orbital period, and $V_{\text {rel }}$ is the relative velocity between the two objects.

Instances of this algorithm have been implemented in the German Long-term Utility for Collision Analysis (LUCA) model [11] as well as the Italian Semi Deterministic Model (SDM) and Aerospace Corporation's ADEPT model. The implementation used for this investigation also includes filters introduced in [11] to reduce the number of pairs considered, by excluding objects in orbits incapable of colliding such as non-proximal objects in synchronised orbits.

\section{CUBE IMPLEMENTATION}

The Cube algorithm was implemented to better understand the behaviour of the model. The results reported by [17, 18] for collision rates between pairs of Jovian moons were used to verify the new implementation. An initial value of $1 \%$ of the average semi-major axis of the moons was chosen for the dimension of the cube, giving side length of $1,150,750 \mathrm{~km}$. The algorithm was then used to repeatedly sample the system to allow average collision rates to be calculated for each collision pair.

The values for the semi-major axis, eccentricity, and inclination, were assumed to be fixed to the values stated by Kessler [18] for the duration of the simulation. However, in addition to random sampling of the mean anomaly the values for the right ascension and argument of perigee were also randomly sampled from a uniform distribution between zero and two pi at each step using the assumption that they could be considered uniformly distributed over the duration of the simulation.

Table 2. Comparing the collision rates $\left(10^{-10} /\right.$ year) calculated by different models.

\begin{tabular}{lllll}
\hline Collision Pair & Kessler (1981) & Kessler (2003) & Liou et al. (2003) - Cube & Our Result - Cube \\
\hline Himalia-Elara & 4.3 & 4.1 & 4.2 & 4.22 \\
Himalia-Lysithea & 2.8 & 3.4 & 3.5 & 3.58 \\
Himalia-Leda & 3.1 & 3 & 3.2 & 3.22 \\
Elara-Lysithea & 0.52 & 0.51 & 0.52 & 0.528 \\
Elara-Leda & 0.57 & 0.57 & 0.56 & 0.592 \\
Lysithea-Leda & 0.039 & 0.038 & 0.038 & 0.0386 \\
\hline
\end{tabular}

The results in Table 2 show that the implementation of the algorithm produced results consistent with those published in previous studies. The convergence of these average collision rates was investigated to understand how many samples were required before the rates appeared to have converged. Samples were taken at 1-day time-steps and the average collision rate calculated every 36,500 steps over 1 million different sets (i.e. total simulation consisted of 36.5 billion samples for each collision pair). 
Figure 4 shows the convergence of the average collision rates, highlighting that convergence of the collision rate for individual collision pairs typically requires a large number samples. After 200,000 sets (7.3 billion applications of the algorithm) there was still considerable variation in the collision rates.

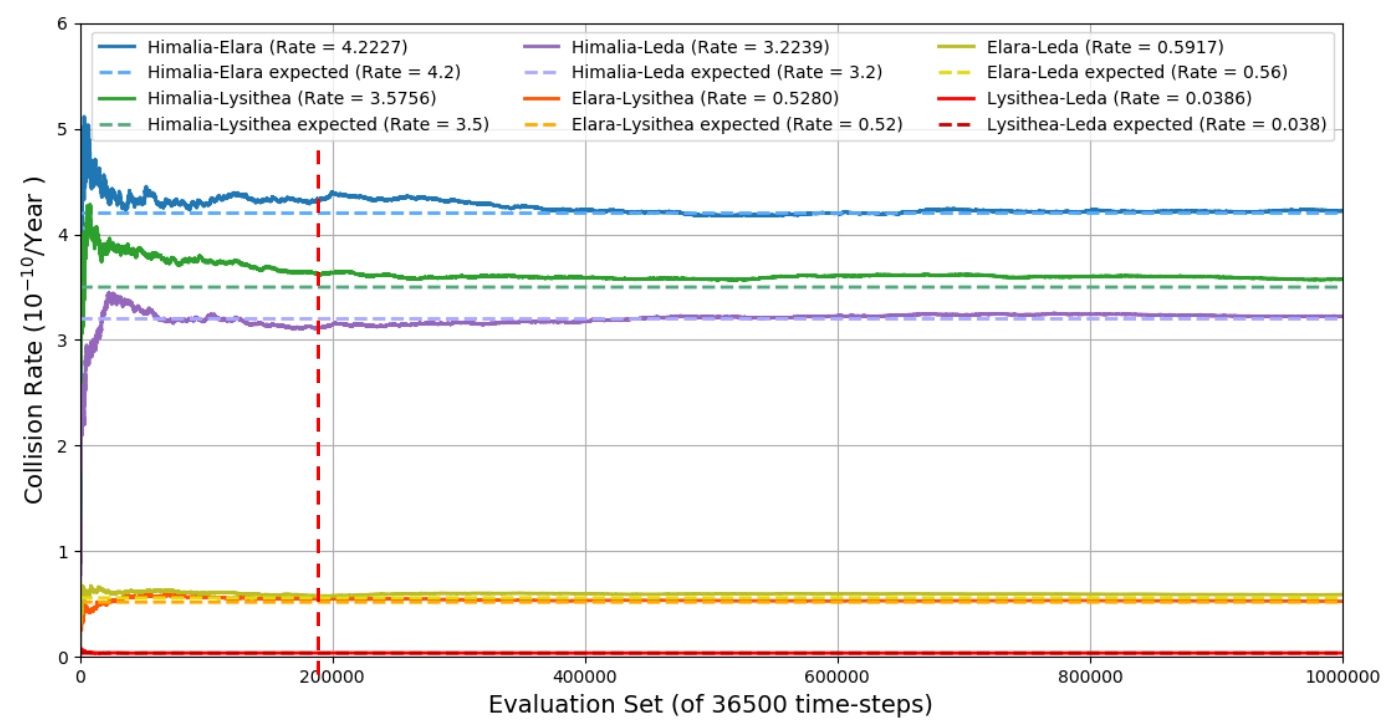

Fig. 4 Convergence of average collision rate for different pairs of Jovian moons compared to the expected final results from previous studies.

By comparison most simulations of the debris environment use 5 day time-steps over 200 years resulting in only 14,610 samples for any specific pair of objects. Additionally, there will be large changes over this time period in the eccentricity and semi-major axis of many of the objects in earth orbit, meaning that fewer of the samples will relate to a particular combination of orbits.

If this rate of convergence holds for debris modelling then the results suggest that a large number of object pairs would be required for confidence in the collision rate produced by the Cube algorithm. This suggests that the number of samples taken in typical space debris simulations is insufficient to form a good approximation of the probability that any specific object will be involved in a collision. However, a sufficiently large object population may provide a good approximation of the environmental collision rate as a whole. Achieving 7.3 billion samples, for example, would require $\sim 499,500$ possible collision pairs, which is a population of 1,000 interacting objects.

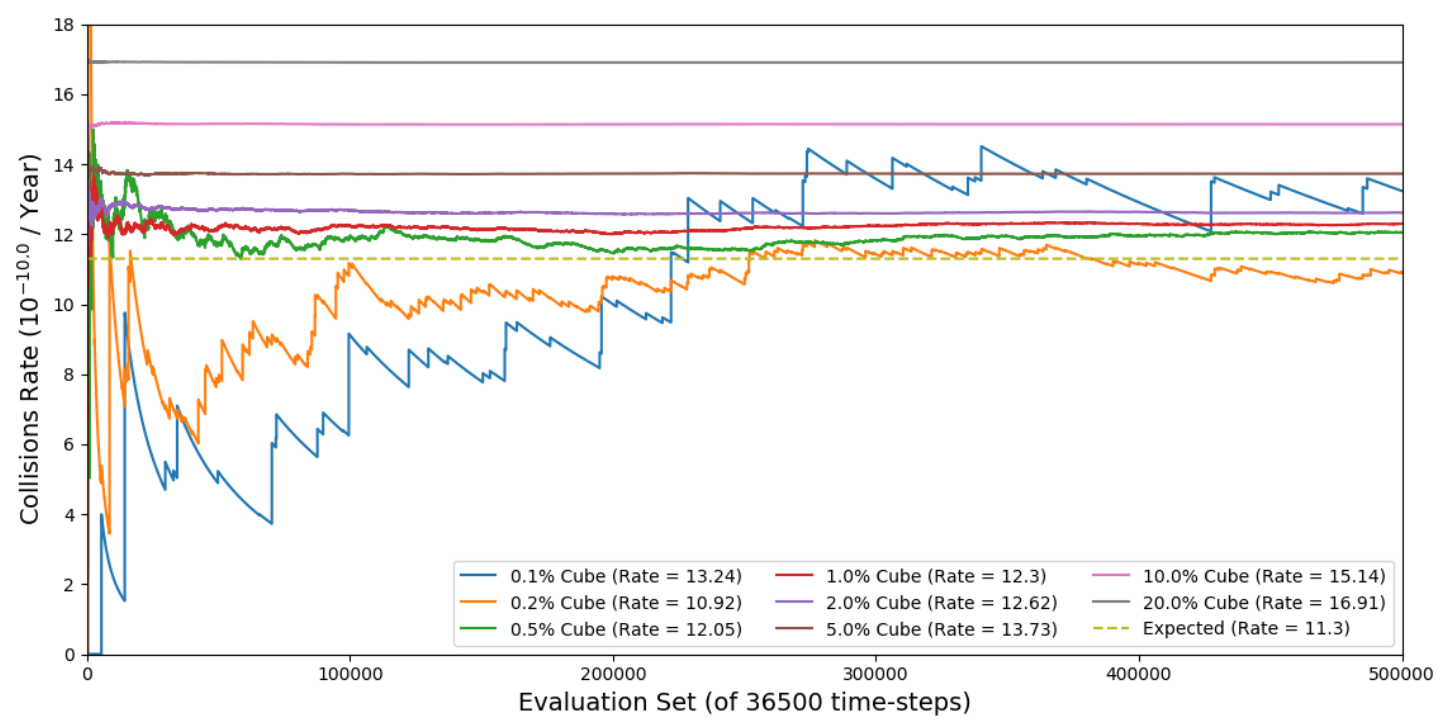

Fig. 5 Convergence and final average rate for the cumulative collision rate of the system for a range of different cube sizes. 
One of the key parameters of the model is the size of the cube. To investigate how the choice of cube dimension affects the performance of the algorithm the Jovian moons scenario was repeated using a range of different sized cubes. Values of $0.1 \%, 0.2 \%, 0.5 \%, 1 \%, 2 \%, 5 \%, 10 \%$ and $20 \%$ of the average semi-major axis of the system were used to compare the convergence of the cumulative collision rate for all pairs of moons.

The results of this investigation are shown in Fig. 5 including the average collision rate at the end of each simulation. Each simulation was conducted using the same seed value for the random number generator to ensure that there was no deviation in the sampling of position for each moon. These results indicate that not only is the rate of convergence dependent on the size of cube used, but also the converged value of the collision rate. Smaller cubes result in a lower average collision rate and require more steps to converge, as is particularly visible in the relatively larger jumps seen in the average collision rate for the $0.1 \%$ cube. The consequence is that the size of the cube used in the model has close to a linear relationship with the estimated collision rates (excluding the rates for the $0.1 \%$ and $0.2 \%$ cubes which did not converge).

In this scenario there are two additional degrees of freedom (right ascension and argument of perigee) compared to simulations of debris in Earth orbit. Further work is required to understand the nature of the relationship between the cube size and the collision rates to ensure that appropriate configurations are used when simulating the evolutions of the debris environment.

\section{COMPARISON OF COLLISION ALGORITHMS}

Different configurations of the Cube and Orbit Trace collision algorithms were compared for several scenarios consisting of sub-sets of the tracked population of objects in Earth orbit. For the Cube these configurations were defined by using a range of sizes of cube.

For the purpose of this investigation two scenarios were chosen by reviewing the backlog of conjunctions reported by Satellite Orbital Conjunction Reports Assessing Threatening Encounters in Space (SOCRATES) [19]. To minimize the computational effort required scenarios were identified from SCORATES where a single primary object had a high number of conjunctions with a relatively small secondary population.

The SOCRATES methodology uses the SGP4 propagator in STK to identify satellite conjunctions with a minimum miss distance of less than $5 \mathrm{~km}$. For the object radii these scenarios followed the SOCRATES standard of using a default hard-body radius of one metre and a database ${ }^{1}$ of the radii used for historic objects.

Table 3. Details of test cases used for comparing collision algorithms.

\begin{tabular}{cccccc}
\hline Primary Object & $\begin{array}{c}\text { Semi-major axis } \\
\text { (km) }\end{array}$ & $\begin{array}{c}\text { Secondary } \\
\text { Count }\end{array}$ & Start Date & $\begin{array}{c}\text { Duration } \\
\text { (days) }\end{array}$ & $\begin{array}{c}\text { Conjunction Frequency } \\
\text { (per day) }\end{array}$ \\
\hline 42731 & 6774 & 46 & $2017-06-02$ & 519 & 0.615 \\
49300 & 6865 & 83 & $2018-12-28$ & 70 & 4.462 \\
\hline
\end{tabular}

Table 3 describes the features of the selected test cases. For each of these scenarios the positions of the objects were initialised using TLEs with epoch times closest to the first reported conjunction in the SOCRATES data. The behaviour of the population was then simulated until after the final reported conjunction using a time-step of 0.05 days ( once per orbit).

\subsection{SGP4 Propagator}

Performing these test cases required the propagation of the orbits of the different objects for the duration of the simulation. An existing C++ implementation of the SGP4 propagator [20] was integrated with the collision algorithms and verified against the test cases defined for the propagator.

Across the 33 test cases the average deviation from the stated results for position and velocity was found to be $4.9 \times 10^{-5} \mathrm{~km}$ and $4.8 \times 10^{-7} \mathrm{~km} / \mathrm{s}$ with standard deviations of $5.2 \times 10^{-5}$ and $1.3 \times 10^{-7}$ respectively.

${ }^{1}$ Dr. T. S. Kelso, the creator of SOCRATES, provided a list of these historic radii, which allowed consistency with the SOCRATES results. 


\subsection{Results}

The probability of collision calculated by each of the collision algorithm configurations was then compared to the collision probabilities predicted in the SOCRATES reports. A comparison of the results of the simulations using different configurations of the Cube and Orbit Trace algorithms can be seen in Table 4 and Table 5 for objects 42731 and 43900 respectively. For simulations using the Cube algorithm the results have been averaged over 10 different simulations.

The results in these tables compare how three different metrics vary across the different configurations of the collision algorithms. The chosen metrics were the number of conjunctions detected, the total combined probability of at least one collision occurring over the entire simulation, and the average collision probability across all conjunctions.

Table 4. Results of different collision algorithms for object 42731 with 47 secondary objects over 519 days

\begin{tabular}{llccc}
\hline Method & Cube Size/ Threshold & $\begin{array}{c}\text { Detected } \\
\text { Conjunctions }\end{array}$ & $\begin{array}{c}\text { Collision } \\
\text { Probability }\end{array}$ & $\begin{array}{c}\text { Average Collision } \\
\text { Probability }\end{array}$ \\
\hline SOCRATES & $5 \mathrm{~km}$ & 320 & $4.25 \mathrm{E}-03$ & $4.61 \mathrm{E}-06$ \\
Orbit Trace & (Hard Body Radius) & 104 & $6.66 \mathrm{E}-06$ & $6.40 \mathrm{E}-08$ \\
CUBE* & $1 \mathrm{~km}$ & 3.1 & $8.94 \mathrm{E}-06$ & $2.34 \mathrm{E}-06$ \\
CUBE* & $2 \mathrm{~km}$ & 18.5 & $7.70 \mathrm{E}-06$ & $4.07 \mathrm{E}-07$ \\
CUBE* & $5 \mathrm{~km}$ & 136.1 & $1.16 \mathrm{E}-05$ & $8.58 \mathrm{E}-08$ \\
CUBE* & $10 \mathrm{~km}$ & 596 & $7.51 \mathrm{E}-06$ & $1.25 \mathrm{E}-08$ \\
CUBE* & $20 \mathrm{~km}$ & 2175.4 & $5.46 \mathrm{E}-06$ & $2.51 \mathrm{E}-09$ \\
CUBE* & $50 \mathrm{~km}$ & 9992.2 & $3.31 \mathrm{E}-06$ & $3.31 \mathrm{E}-10$ \\
CUBE* & $100 \mathrm{~km}$ & 27585.6 & $2.04 \mathrm{E}-06$ & $7.39 \mathrm{E}-11$ \\
\hline
\end{tabular}

* For the cube examples results are averaged over 10 runs

The most obvious result visible in the tables is that the overall collision probability is consistent for Orbit Trace and the different Cube configurations. However, the number of conjunctions and the average collision probability vary by several orders of magnitude. Comparing with the SOCRATES results, both the overall and average collision probability of all configurations were smaller by several orders of magnitude.

The relationship between the SOCRATES results and the various configurations was inconsistent between the two test cases. As expected the number of conjunctions detected increased with increasing threshold and cube size. However, where the closest number of conjunctions to the SOCRATES data was with a $5 \mathrm{~km}$ cube for the first scenario it was with a $10 \mathrm{~m}$ threshold and $20 \mathrm{~km}$ cube for the second scenario. The number of conjunctions recorded for a $5 \mathrm{~km}$ cube size is comparable to Orbit Trace for both scenarios.

Table 5. Results of different collision algorithms for object 43900 with 83 secondary objects over 70 days

\begin{tabular}{llccc}
\hline Method & Cube Size/ Threshold & $\begin{array}{c}\text { Detected } \\
\text { Conjunctions }\end{array}$ & $\begin{array}{c}\text { Collision } \\
\text { Probability }\end{array}$ & $\begin{array}{c}\text { Average Collision } \\
\text { Probability }\end{array}$ \\
\hline SOCRATES & $5 \mathrm{~km}$ & 218 & $1.20 \mathrm{E}-03$ & $1.14 \mathrm{E}-06$ \\
Orbit Trace & (Hard Body Radius) & 20 & $4.75 \mathrm{E}-06$ & $2.38 \mathrm{E}-07$ \\
CUBE* & $1 \mathrm{~km}$ & 0.67 & $5.15 \mathrm{E}-07$ & $3.82 \mathrm{E}-07$ \\
CUBE* & $2 \mathrm{~km}$ & 4.8 & $6.82 \mathrm{E}-07$ & $1.48 \mathrm{E}-07$ \\
CUBE* & $5 \mathrm{~km}$ & 25.1 & $3.72 \mathrm{E}-07$ & $1.47 \mathrm{E}-08$ \\
CUBE* & $10 \mathrm{~km}$ & 70.5 & $6.60 \mathrm{E}-06$ & $9.52 \mathrm{E}-08$ \\
CUBE* & $20 \mathrm{~km}$ & 163.3 & $4.17 \mathrm{E}-06$ & $2.57 \mathrm{E}-08$ \\
CUBE* & $50 \mathrm{~km}$ & 477.1 & $3.49 \mathrm{E}-06$ & $7.32 \mathrm{E}-09$ \\
CUBE* & $100 \mathrm{~km}$ & 1131.4 & $2.11 \mathrm{E}-06$ & $1.87 \mathrm{E}-09$ \\
\hline * For the Cube examples results are averaged over 10 runs & \\
\hline
\end{tabular}


The collision probabilities achieved with the different cube sizes are all within an order of magnitude, with any trend observed being a tendency for the probability to decrease for increasing cube size (excluding scenarios with few conjunctions), in contrast to the results of the investigation into the Jovian moons scenario. While the number of conjunctions increased proportional to cube size the overall collision probability was inversely proportional to the number of conjunctions, The result was that there was not a large change in the overall collision probability with increasing cube size. The increased probability of conjunction that comes with increased cube size is also likely to result in these conjunctions involving a wider range of objects. This suggests that while changing the size of the cube may not have a large impact on the number of collisions in simulations of the debris environment, it may change which pairs of objects experience collisions.

Each of the methods was used to quantify the risk of a collision occurring but provided a very different assessment of the risk. This suggests that there is currently a poor level of understanding of the rates at which collisions can be expected to occur in the future.

Looking specifically at NewSpace systems there are several issues when applying the Cube algorithm. In particular, when modelling large constellations there are issues with the approach of sampling from the mean anomaly. This randomization of the position is not consistent with the structured relationship of the constellation orbits. This is likely to result in over-prediction of collision rates within a constellation when modelled as independent objects.

The trends in the characteristics of spacecraft include the observed shift towards a higher proportion of spacecraft in lower orbits as well as a trend towards smaller spacecraft, such as CubeSats. The positional uncertainty is larger for these small spacecraft and for spacecraft in lower altitudes where the atmospheric drag force is greater. This would suggest that a larger cube size might be required due to the greater positional uncertainty.

However, the choice of cube size also impacts the suitability of the application of the kinetic theory of gas within the cube. The collision rate in Eq. 2 is dependent on the collision cross section of the objects and is agnostic to the minimum separation. As a result, increased cube size would increase the number of conjunctions observed between large objects in more separated orbits which would be assigned a higher probability of collision than between smaller more closely interacting objects.

It is possible that the Cube algorithm, with its speed benefits, can be used to look at the overall environmental hazard, even though it is unsuitable for studying individual object pairs. However, the issues raised by this investigation creates doubts on the applicability of the results of simulations of the debris environment. A change in which objects are likely to be involved in collisions could substantially alter how many and at what altitude fragments are generated which in turn impacts the life-time of the fragments produced and the collision risk they pose to other objects. This could result in long term impacts on the evolution of the debris environment.

\section{CONCLUSIONS}

The commercial sector has grown over the last 40 years and now contributes more than half of new spacecraft launched. As well as changes in the average mass and cross-section of spacecraft this shift has been accompanied by changes in the concepts of spacecraft deployment and operation. One result of these changes is an increase in the clustering of spacecraft into specific inclination bands and lower altitudes.

A study of the results of the Cube for the original Jovian moons scenario highlighted that a substantial variation existed in the average collision rate for an individual object pair after 7 billion samples. For standard simulations of the debris environment consisting of only 14,610 samples in time these results suggest that the Cube method is not suitable for estimating the collision probability of a specific pair of objects. Further investigation showed that the rate of convergence and the overall collision probability of the test case was dependent upon the choice of cube size. The results showed that for smaller cubes a greater number of samples was required for convergence and indicated a linear relationship of decreasing collision rate for decreasing cube size.

A comparison of different approaches for the more restricted case of spacecraft in earth orbit showed consistency between the total collision probabilities when using each of the Orbit Trace and Cube methods at a level several order of magnitude below that reported by SOCRATES. The relation between the SOCRATES results and the results for the different collision thresholds and cube sizes used did not appear to be consistent between the two scenarios. This questions whether these models provide an accurate representation of the likelihood of collisions in the debris environment.

Larger cube sizes resulted in significantly more conjunction events, each with a lower collision probability. However, in contrast with the earlier investigation the overall collision probability appeared to increase with 
decreasing cube size for these scenarios. A comparison with the results of the Orbit-Trace method suggests the use of cube sizes in the range 5-20 km, but the exact size varied between the two scenarios.

This suggests that the Cube may introduce a slight bias towards collisions within certain sub-sets of the debris population. Further work is required to investigate the discrepancies between the different algorithms. Future work is also planned to determine how the results vary when NewSpace systems such as constellations are introduced.

\section{ACKNOWLEDGEMENTS}

The authors acknowledge support from the University of Southampton EPSRC Centre for Doctoral Training in Next Generation Computational Modelling (Grant No. EP/L015382/1). The authors would also like to thank ESA for providing downloads of spacecraft data from the DISCOS database and Dr. T.S. Kelso of CelesTrak for providing historical values for object radii.

\section{REFERENCES}

1. European Space Agency (ESA), Space debris by the numbers, January 2019, https://www.esa.int/ Our_Activities/Operations/Space_Debris/Space_debris_by_the_numbers, (accessed 23.09.19).

2. Beck, J. Review of Space Debris Population Prediction Methodologies and Results, Belstead Research Ltd., Waterlooville, UK, Rep. no. PR00003/D02, November 2013.

3. Kessler, D.J. and Cour-Palais, B.G. Collision Frequency of Artificial Satellites: The Creation of a Debris Belt, Journal of Geophysical Research, 83(A6), 2637- 2646.

4. Kessler, D.J. and Anz-Meador P.D., Critical number of spacecraft in low Earth orbit: using satellite fragmentation data to evaluate the stability of the orbital debris environment, Proceedings of the Third European Conference on Space Debris, 2001, 19 - 21 March.

5. Bryce Space and Technology, Start-Up Space: Update on Investment in Commercial Space Ventures 2018, https://www.brycetech.com/downloads/Bryce_Start_Up_Space_2018.pdf (accessed 18.01.2019).

6. Sudhakar, G.P. ISRO: 104 Satellites in 1 go, Vidyaniketan Journal of Management Research, Proceedings of International Conference on Strategy, System and Services for Sustainability and Scalability of Business (5S), School of Management, Pondicherry University, Pondicherry, India, 2018, March 23-24.

7. Paikowsky, D. What Is New Space? The Changing Ecosystem of Global Space Activity. New Space, 5(2), (2017) 84-88.

8. Henry, C. SpaceX plans 24 Starlink launches next year, September 10 2019, https://spacenews.com/spacexplans-24-starlink-launches-next-year/ (accessed 24-09-19).

9. Johnson, N.L., et al. NASA's new breakup model of EVOLVE 4.0, Advances in Space Research, 28(9), (2001), 1377-1384.

10. Liou, J. C. Collision activities in the future orbital debris environment, Advances in Space Research, 38(9), (2006), 2102-2106.

11. Radtke, J., et al. LUCA2 - An enhanced long-term utility for collision analysis, Proceedings of the 7th European Conference on Space Debris, Darmstadt. Germany, 2017, 18 - 21 April.

12. Hoots, F.R. and Roehrich, R.L. Spacetrack Report No. 3--Models for Propagation of NORAD Elements Sets, Spacetrack Report, 3(3), (1980) 1-91.

13. Anz-Meador, P. D., et al. History of On-Orbit Satellite Fragmentations, 15th Edition, Orbital Debris Program Office, NASA, Houston, Texas, NASA/TM-2018-220037, July 2018.

14. Öpik, E. J. Collision Probabilities with the Planets and the Distribution of Interplanetary Matter, Proceedings of the Royal Irish Academy. Section A: Mathematical and Physical Sciences, 54:165-199.

15. Wetherill, G. W. Collisions in the asteroid belt. Journal of Geophysical Research, 72(9), 2429-2444, 1967. https://doi.org/10.1029/JZ072i009p02429

16. JeongAhn, Y., and Malhotra, R. Simplified Derivation of the Collision Probability of Two Objects in Independent Keplerian Orbits. The Astronomical Journal, 153(5), 235, 2017. https://doi.org/10.3847/15383881/aa6aa7 
17. Liou, J.-C., et al. A New Approach to Evaluate Collision Probabilities among Asteroids, Comets. Lunar and Planetary Science, XXXIV: 2-3, 2003.

18. Kessler, D.J. Derivation of the collision probability between orbiting objects: the lifetimes of Jupiter's outer moons. Icarus, 48(1):39-48, 1981

19. Kelso, T.S. and Alfano, S. Satellite Orbital Conjunction Reports Assessing Threatening Encounters in Space (SOCRATES), Proceedings of the 4th European Conference on Space Debris. Darmstadt, Germany, 2005.

20. Vallado, D., et al. P. Revisiting Spacetrack Report \#3, AIAA/AAS Astrodynamics Specialist Conference and Exhibition Reston, Virginia, 2006. 